und jun. mittels der galvanocanstischen Schlinge die Vaginalportion ab. Auch hier war der Erfolg, trotzdem das Fibroid vollständig intact blieb, ein höchst zufriedenstellender. Auch jetzt, nach 2 Jahren, ist der Gesundheitszustand nach den mir zugehenden Berichten, gegenüber der früheren Zeit, ein sehr günstiger.

\title{
Zum Verhalten des Scheideneinganges nach der Geburt.
}

\author{
Vorläufige Mittheilang \\ von
}

E. Bellien, stud. med, in Charkow.

Bei den Untersuchungen des Verhaltens des Hymen in seinen verschiedenen physiologischen Zuständen, respective des Verhaltens seiner Reste nach der Geburt, welche ein Material von 286 Exemplaren betreffen, die ich in hiesiger geburtshülflichen Klinik auszuführen die Möglichkeit hatte, gelangte ich zu Resultaten, welche mir der Mittheilung nicht unwerth erscheinen; dieselben sind theils Bestätigung des schon Bekannten, theils keziehen sie sich auf neuergründete Thatsachen - die Entstehungsweise der Carunculae myrtiformes betreffend.

Vorliegende Mittheilung betrifft nur die Veränderungen des $\mathrm{Hy}_{y}$ men nach der Geburt.

Sehröder in Erlangen hat in letzterer Zeit mehrere Male darauf aufmerksam gemacht (in ,Sehwangerschaft, Geburt und Wochenbett". 1867. \$. 6., in der Gynäkologischen Section der Versammlung dentseher Naturforscher und Aerzte in Rostock. 1871. Sitzung 18. September), dass die bisherigen Vorstellungen vom Hymen und den Carunculae martiformes nieht ganz zutreffend sind. Seiner Meinung nach werden die Carunculae myrtiformes nie vor der ersten Geburt beobachtet; ihre Entstehung verdanken dieselben einer blutigen Suffundirung und späteren im Wochenbette eintretenden Gangränescenz der einzelnen Lappen des Hymen, welche dureh den Druck, dem dieselben beim Durchschneiden des Kindskopfes ausgesetzt sind, bedingt wird. Meine Beobachtungen erlauben mir die Richtigkeit der ersten Meinung vollständig zu constatiren; nie habe 
ich bei Frauen, welche nie geboren haben, charakteristische fleischige, auf breiter Basis sitzende, konische oder warzenförmige Carunculae myrtiormes vorgefunden; der ganze Unterschied dieser deflorirten $\mathrm{Hy}$ menes bei Frauen, welche noch nicht geboren hatten, von virginalen bestand nur in stellenweisen Einrissen und einiger Verdickung der gebildeten Lappen, welche nichts Identisches mit den Carunculae myrtiformes darstellten.

Jedoch die Entstehungsweise dieser eine überstandene Geburt bestimmenden Carunculae myrtiformes glaube ich am allerwenigsten der Suffundirung and späteren Gangränescenz der Hymenlappen zuschreiben zu dürfen; vielmehr haben mich parallele Beobachtungen des Scheideneinganges for und nach der Geburt von einer anderen Entstehungsweise übexzengt, welche von Herrn Prof. Lazarewits ch in seinen klinischen Vorträgen seit einiger Zeit hypothetisch gelehrt wird. Das beständige Vorfinden der charakteristischen Carunculae myrtiformes im unteren Theile des Scheideneinganges brachte auf den Gedanken, dass dieselben ihre Entstehung den Rändern eines tiefen Einrisses, welcher die Basis des Hymen und das unter ihr liegende Gewebe - den äusseren Rand der Scheidenwand betrifft, verdanken. Meine Beobachtnngen ergaben nun: fast in allen Fällen, wo vor der Geburt (bei Primiparen) die Continuität des Hymen erhalten war oder nur einzelne Einrisse darbot, wie es meistentheils der Fall ist, fand ich nach der Geburt im hinteren oder unteren Theile des Scheideneinganges Einrisse, welche nicht nur den Hymen, sondern auch seine Basis - die unter ihm liegenden Gewebe betraf. Die Winkel solcher Einrisse verwandelten sich nach und nach in charakteristische konische oder warzenförmige, auf breiter Basis sitzende Carunculae myrtiformes, die ich in vielen Fällen schon am 7.-9. Tage post partum genau als solehe constatiren konnte. Im oberen Theile des Scheideneinganges habe ich nie charakteristische Carunculae vorgefunden; die Veränderung des oberen Hymentheiles bestand nach der Geburt nur in Einrissen desselben, einiger Verdickung der Lappen und Abrundung der Lappenränder. In einigen, iibrigens höchst exclusiven Fällen fand ich nach der Geburt gar keine Carunculae vor, konnte aber zugleich nur Einrisse, welche den Hymen selbst, nicht aber das unter ihm liegende, aus dickem Binde- und Muskelgewebe bestehende Gewebe betrafen, constatiren. Ich kann also durch meine Beobachtungen die Richtigkeit der Meinung des Herrn Prof. Lazarewitsch, nach welcher die dicken auf breiter Basis placirten charakteristischen Carunculae myrtiformes ihre Entstehung nicht dem Hymen selbst, sondern dem dicken Bindegewebe seiner Basis verdanken, vollständig bestätigen. Hierdurch wird denn auch die Dicke und die breite Basis der Carunculae myrtiformes erklärlich, da das Gewebe, dem sie ihre Entstehung verdanken, an und für sich schon viel dicker ist, als das Gewebe des Hymen. Was die Defecte in der Fortsetzung des Hymen, welche nach der Geburt oft beobachtet werden, betrifft, so ist, wie ich glaube, ihre Entstehung ebenfalls am wenigsten der Gangränescenz und Zerstörung der Hymenlappen zuzuschreiben; eher möchten dieselben von einer partiellen Abtrennung . 
134 Dohrn, Eine historische. Bemerkung über die Erklärung u. s. w.

des Hymen von seiner Basis herrühren. Fälle, in denen man auf einer Seite einen ziemlichen Defect der Hymensubstanz, auf der anderen Seite dagegen oder daneben einen langen zungenförmigen Lappen vorfindet, bekräftigen dies vollständig

Die ausführliche Beschreibung meiner Beobachtungen wird nach Erörterung noch einiger anderer Fragen erfolgen.

Meine Beobachtungen wurden in der Klinik des Herm Prof. Lazarewitsch ausgefïhrt, dem zugleich hiermit meinen aufrichtigen Dank auszusprechen, ich für die angenehmste Pflicht halte.

\title{
Eine historische Bemerkung über die Erklärung des Zusam- menhanges von Nabelschnurbruch und Atresia ani.
}

\author{
Von \\ Dohrn.
}

Ahlfeld hat im 2. Hefte des 5. Bandes dieses Archivs eine Missbildung mit Nabelschnurbruch und Atresia ani beschrieben und die letztere mit dem seitens der vorgelagerten Därme ausgeübten Zuge in Zusammenhang gebracht. Es ist gewiss in hohem Grade wahrscheinlich, dass diese Erklärung richtig ist; wenn aber Ahlfeld glaubt, dass er der Erste sei, welcher diese Erklärung giebt, so befindet er sich im Irrthum. Der verstorbene Prof. Claudius zu Marburg hat schon vor 9 Jahren die gleiche Erklärung durch einen seiner Schüler, $\nabla$. Roques in Treysa, veröffentlichen lassen. In der Dissertation desselben "Ueber einen menschlichen Acardiacus mit Nabelschnurbruch und Atresia ani, Marburg 1864" steht Seite 16 Folgendes:

„Dieser Process des Entgegenwachsens kann jedoch nur dann stattfinden, wenn er keinerlei Störung erleidet. Was diese Verwachsung wohl hindern könnte, wäre eine Zerrung des Mastdarmes, und diese Zerrung wird hauptsächlich durch einen Nabelbruch hervorgebracht, in welchem jedoch Theile des Dickdarmes sich befinden müssen, denn es leuchtet aus der anatomischen Betrachtung der Dünndärme und deren Befestigung ein, dass ein Liegen desselben in einem Bruchsacke keine Zerrung auf den Mastdarm ausüben könne. Je nach dem Grade der Zerrung kommt nun eine Cloake zu Stande, indem 